ISSN 0103-9954

\title{
RELAÇÃO ENTRE ALONGAMENTO ANUAL DA COPA E O INCREMENTO DIAMÉTRICO DE ÁRVORES SINGULARES DE Cedrela fissilis (Vell)
}

\author{
RELATIONSHIP BETWEEN ANNUAL CROWN ELONGATION AND DIAMETER INCREMENT IN \\ SINGULAR Cedrela fissilis (Vell) TREES
}

\author{
Thiago Augusto da Cunha ${ }^{1}$ Jean Pierre Cavalli ${ }^{2}$ César Augusto Guimarães Finger ${ }^{3}$
}

\begin{abstract}
RESUMO
O crescimento da copa é uma variável pouco conhecida no campo florestal. Esta estrutura fotossintética tão importante para o crescimento da árvore encontra-se em um nível de difícil acesso para o manejador florestal e, portanto, pouco se conhece sobre seu desenvolvimento. Assim, o objetivo dessa pesquisa foi modelar o alongamento anual da copa utilizando como variável independente o incremento corrente anual em diâmetro do tronco de Cedrela fissilis. Utilizando 22 árvores singulares determinou-se o alongamento anual da copa e o incremento corrente anual em diâmetro no período de oito anos. Demonstrou-se que o desenvolvimento da copa esta relacionado com o incremento diamétrico $(\mathrm{r}=0,53 ; \mathrm{p}<0,0001)$, entretanto, para estimar o alongamento anual da copa com maior precisão, o modelo linear misto também incluiu o diâmetro a altura do peito e a altura comercial, que explicou $70 \%$ da variação total do alongamento do galho por classe de altura total.
\end{abstract}

Palavras-chave: crescimento da copa; regressão linear mista; estrutura de covariância.

\begin{abstract}
The annual crown growth is a little known variable in the forestry field. This important photosynthetic structure for tree growth is in a difficult level-access to the forester and therefore, a little knowing about its development. Then, the objective of this research was to model the annual crown growth using as independent variable the diameter at breast height increment of Cedrela fissilis. By using 22 singular ones, we determined the annual crown growth and the current annual diameter increment for the period of eight years. We demonstrated that the crown development are related with the diameter increment $(r=0.53$; $\mathrm{p}<0.0001)$. However, to predict the annual crown elongation with more precision, the linear mixed model also included the diameter at breast height and the commercial height explaining $70 \%$ of the total variation in the crown elongation by total height class.
\end{abstract}

Keywords: crown growth; mixed model; covariance structure.

\section{INTRODUÇÃO}

Os regimes de manejo são compostos por técnicas silviculturais definidas a partir de pesquisas sobre o comportamento das espécies, relacionado ao objetivo da produção. A utilização de regimes de manejo eficientes e pré-determinados pode resultar em uma produção estável, com maior produtividade e melhor qualidade do produto final. Por isso é extremamente importante que o administrador florestal avalie e quantifique o crescimento e a produção de suas florestas, promovendo um planejamen-

1 Engenheiro Florestal, Dr., Professor Adjunto do Centro de Ciências Biológicas e Naturais, Universidade Federal do Acre, Rodovia BR-364, Km 4, Distrito Industrial, CEP 69915-900, Rio Branco (AC). dacunha@ufac.br

2 Engenheiro Florestal, Mestrando do Programa de Pós-Graduação em Engenharia Florestal, Centro de Ciências Rurais, Universidade Federal de Santa Maria, Av. Roraima, 1000, CEP 97105-900, Santa Maria (RS), Brasil. jeancavalli@mail.ufsm.br

3 Engenheiro Florestal, Dr., Professor Associado do Departamento de Ciências Florestais, Centro de Ciências Rurais, Universidade Federal de Santa Maria, Av. Roraima, 1000, CEP 97105-900, Santa Maria, (RS), Brasil. caesar.finger@gmail.com

Recebido para publicação em 27/12/2010 e aceito em 01/08/2012 
to criterioso da produção através da prescrição de regimes de manejo adequados, visando à qualidade do produto final (ACERBI et al., 2002; EISFELD et al., 2005).

O desenvolvimento de pesquisas geralmente está voltado para espécies de alto valor de mercado e que possuam potencial para o estudo dendrocronológico, sendo de grande relevância, principalmente em regiões onde os recursos florestais naturais encontram-se reduzidos a fragmentos. Em fragmentos florestais, nas regiões sul e sudeste do Brasil, é comum a presença de espécies arbóreas com grande potencial científico e econômico, por exemplo, Cedrela fissilis (cedro). As propriedades e características da madeira de cedro a tornam economicamente importante devido a sua ampla utilização na indústria madeireira, e dendrocronologicamente valorizada por possuir anéis de crescimento anuais e distintos (BAPTISTA, 2002).

Algumas pesquisas de relações de tamanho da árvore de cedro e outras espécies nativas foram conduzidas como, por exemplo, a modelagem da forma da copa (DURLO et al., 2004), morfometria da árvore (DURLO e DENARDI, 1998) e características da arquitetura da copa e do galho (REMPHREY e PEARN, 2002). Entretanto, pesquisas sobre o crescimento anual da copa e sua relação com outras variáveis não foram realizadas até o momento em árvores singulares de cedro. A grande motivação por investigar variáveis em árvores livres de concorrência é a possibilidade de estabelecer parâmetros determinantes para as intervenções silviculturais, quando as árvores estiverem crescendo em maciços florestais.

Assim, o conhecimento da alometría de árvores que se desenvolvem com total disponibilidade de luz tem sido útil na tomada de decisão para o manejo de plantios comerciais e, principalmente, de florestas mistas inequiâneas, a partir de modelos de crescimento de árvores individuais capazes de simular o crescimento em povoamentos mistos inequiâneos (MITCHELL 1975; PRETZSCH 1992; PRETZSCH, 1995). Estes modelos geralmente consideram variáveis de copa e suas relações, como preditoras do crescimento das árvores (HEMERY et al., 2005; DURLO et al., 2004), pois são responsáveis pelo aproveitamento da radiação solar, além de refletir um nível cumulativo de competição no tempo (HASENAUER; MONSERUD, 1996), e permite estimar o espaço vital de uma árvore (NUTTO, 2001). Conforme Hemery et al. (2005), o conhecimento das relações entre a copa e o diâmetro do tronco pode ser utilizado na definição de regimes de desbastes, determinação de espaçamentos em plantios mistos ou sistemas agroflorestais, definição de tratamentos silviculturais como a liberação em florestas naturais, seleção genética e na modelagem do crescimento das árvores.

A espécie Cedrela fissilis é decídua, possui características como crescimento radial rítmico, o que favorece a pesquisa desenvolvida a partir de técnicas dendrocronológicas. Espécies que apresentam este tipo de crescimento tendem à formação de extensões de brotos anuais, os quais conduzem o crescimento da copa (WORBES, 1995). Para o cedro, estas extensões são facilmente identificadas por cicatrizes podendo ser contadas. A existência de extensão anual na copa foi comprovada pela relação entre o número de anéis de crescimento da base de cada galho com o número de extensões do respectivo galho (p.e. WORBES, 1995; MATTOS et al., 1999). Neste sentido, o objetivo dessa pesquisa foi modelar o alongamento anual da copa em função do crescimento em diâmetro do tronco de árvores singulares de Cedrela fissilis. Esta pesquisa esta direcionada para contribuir com informações para gerar diretrizes para atividades silviculturais da espécie.

\section{MATERIAIS E MÉTODOS}

\section{Área de estudo}

Um total de 22 árvores singulares de cedro foram amostradas de forma aleatória numa área situada entre as coordenadas $29^{\circ} 43^{\prime} 57^{\prime \prime}$ e $29^{\circ} 55^{\prime} 30^{\prime \prime}$

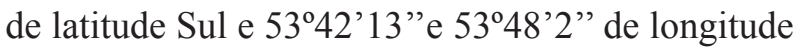
Oeste no Município de Santa Maria, RS. Em termos climáticos, a região de Santa Maria se classifica como do tipo "Cfa 2", subtropical úmido, de acordo com a classificação de Köppen, caracterizado por apresentar uma temperatura do mês mais frio variando de -3 a $18^{\circ} \mathrm{C}$. O regime de chuvas da região é uniformemente distribuído durante todo o ano, com precipitações variando entre os limites de 109 a $177 \mathrm{~mm}$, com média anual de 1691 (MORENO, 1961).

\section{Alongamento do galho (AG)}

Em cada árvore, a copa foi dividida em quatro raios imaginários com ângulos constantes de $90^{\circ}$ e em cada raio extraído um galho de forma que seu comprimento total permitisse a contagem de, no mínimo, oito extensões anuais de crescimento da copa 
(cada um denominado de alongamento do galho (AG) conforme a Figura 1).

Ao longo de toda a extensão de cada galho, cada AG foi identificado e medido com fita métrica e suas médias anuais, para cada árvore, utilizadas na modelagem. Para checar a acurácia da determinação do AG, um disco na base de cada galho foi extraído e realizado a sincronização do número de anéis com o respectivo número de extensões anuais (p.e. WORBES, 1995).

\section{Crescimento corrente anual em diâmetro (ICAd)}

Dois rolos de incremento foram extraídos ao nível do diâmetro a altura do peito (dap) de cada árvore, utilizando trado Pressler, e fixados em suporte de madeira para preparação com estilete profissional e micrótomo de deslizamento.

O incremento corrente anual em diâmetro (ICAd), para o período avaliado, foi medido com precisão de 1/100 mm em mesa micrométrica Lintab II (Rinntech, Heidelberg, Alemanha), e registrado no software TSAP-Win (Rinn, 2003).

\section{Variáveis dendrométricas}

Variáveis de tamanho da árvore foram coletadas seguindo o modelo da árvore proposto por Burger (1939). Estas variáveis incluíram o diâmetro a altura do peito (d) em centímetros; altura do fuste (hc) em metros; altura total (h) em metros; comprimento de copa (1) em metros; raio da copa em metros medido ao longo de oito raios com ângulos horizontal constantes de $45^{\circ}$. O raio médio foi utilizado para estimar o diâmetro da copa (dc). Para a obtenção das medidas utilizou-se fita diamétrica, hipsômetro Vertex III e bússola.

\section{Análise estatística}

O coeficiente de correlação de Pearson foi usado para avaliar a correlação em série dos dados de alongamento da copa (autocorrelação do crescimento), na hipótese de o alongamento do ano "i" depender do alongamento do ano "i-1".

A estratégia de análise empregou um modelo linear misto capaz de modelar a estrutura de dependente dos dados e descrever o i-ésimo valor do AG em função do incremento em diâmetro (Tabela 1, Equação 1). Nesta equação o intercepto e coeficiente de regressão angular (ICAd) foram aleatorizados de forma que os mesmos variassem entre as classes de altura total, previamente estabelecidas.

As variáveis dendrométricas foram incluídas na análise como covariáveis para avaliar o efeito do tamanho inicial da árvore sobre o alongamento do galho (Tabela 1, Equação 2). Durante a análise, covariáveis não significantes foram removidas da equação.

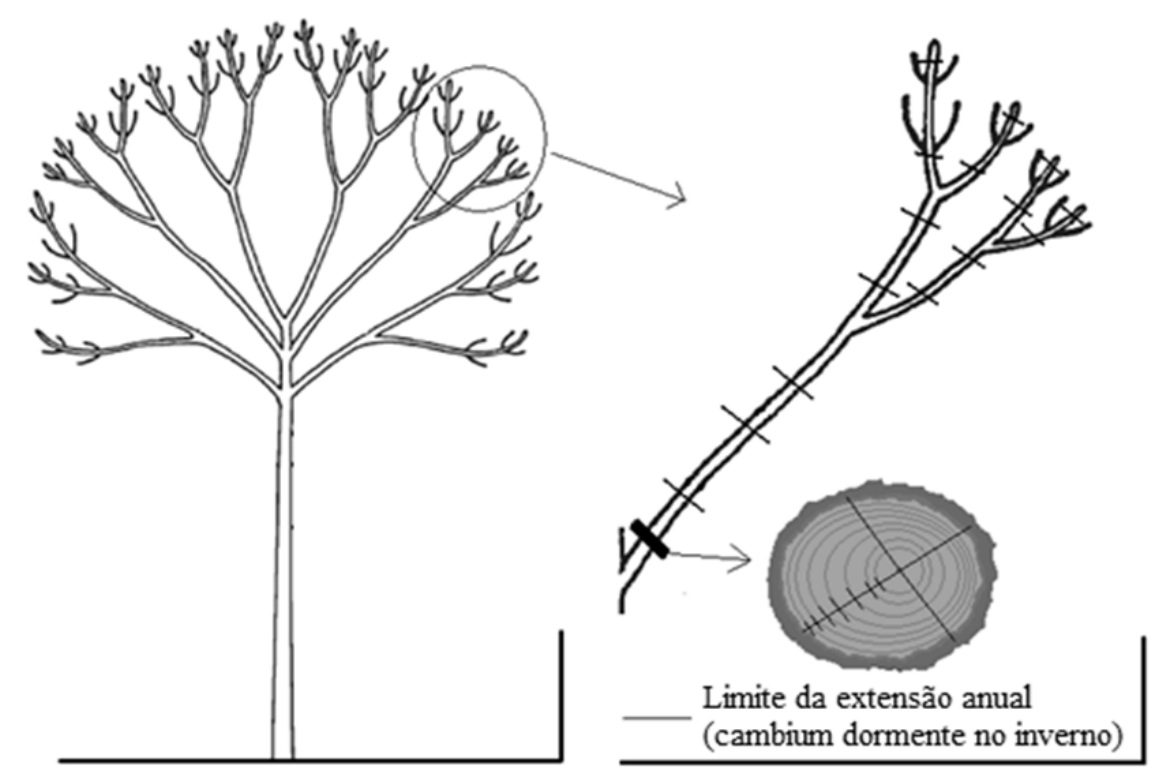

FIGURA 1: Esquema de medição do alongamento do galho de Cedrela fissilis, destacando as extensões de crescimento do galho (incremento anual) e o disco transversal retirado na base do galho.

FIGURE 1: Measure of crown growth of Cedrela fissilis, stressing crown growth units (annual increment) and a transversal disc from the base of branch. 
TABELA 1: Modelos para descrever o alongamento do galho de Cedrela fissilis.

TABLE 1: Models to describe crown growth of Cedrela fissilis trees.

$$
\begin{aligned}
& \qquad A C_{i j}=\beta_{0 j}+\beta_{1 j} \cdot I C A d_{i j}+\varepsilon_{i j}, \\
& \beta_{0 j}=\gamma_{00}+u_{0 j} \\
& \beta_{1 j}=\gamma_{10}+u_{1 j} \\
& \therefore \quad \\
& A C_{i j}=\left[\gamma_{00}+\gamma_{10} \cdot I C A d_{i j}\right]+\left[u_{0 j}+u_{1 j} \cdot I C A d+\varepsilon_{i j}\right]
\end{aligned}
$$

$$
A C_{i j}=\beta_{0 j}+\beta_{1 j} \cdot I C A d_{i j}+\varepsilon_{i j},
$$

Em que:

$\beta_{0 j}=\gamma_{00}+\gamma_{01} \cdot x_{k i j}+u_{0 j}$

$\beta_{k j}=\gamma_{k 0}+\gamma_{k 1} \cdot x_{k i j}+u_{1 j}$

$\therefore$

$$
\begin{aligned}
& A C_{i j}=\left[\gamma_{00}+\gamma_{10} \cdot I C A d_{i j}+\gamma_{k 1} \cdot x_{k i j}+\gamma_{k 1}\left(\operatorname{ICAd}_{i j} \cdot x_{k i j}\right)\right] \\
& +\left[u_{0 j}+u_{1 j} \cdot I C A d_{i j}+\varepsilon_{i j}\right]
\end{aligned}
$$

Em que: $\mathcal{E} \sim N(0 ; R) ;\left(\begin{array}{l}u_{0 j} \\ u_{1 j}\end{array}\right) \sim N(0 ; G) \mathrm{k}=$ número de covariáveis incluídas no modelo; $\mathrm{i}=$ ano de medição; $\mathrm{j}=$ classe de altura total; $\mathrm{x}=$ covariáveis (diâmetro, altura, relação hd, comprimento e diâmetro de copa).

Os efeitos aleatórios associados aos coeficientes de regressão $\left(u_{0 j}+u_{l j} I C A d_{i j}\right)$ foram avaliados utilizando matriz de covariância não estruturada (Equação 3) e para o componente erro residual $\left(\varepsilon_{i j}\right)$ utilizou-se a matriz de covariância com estrutura autorregressiva heterogênea de $1^{\mathrm{a}}$ ordem (Equação 4) que considera um declínio exponencial da correlação $(\rho)$ e variâncias heterogêneas (diagonal principal) entre as observações repetidas ao longo do tempo.

$$
\mathrm{a} G=\left[\begin{array}{ll}
\sigma_{00}^{2} & \sigma_{10} \\
\sigma_{10} & \sigma_{11}^{2}
\end{array}\right]
$$

$$
R=\left[\begin{array}{cccccccc}
\sigma_{1}^{2} & & & & & & \\
\sigma_{2} \sigma_{1} \rho & \sigma_{2}^{2} & & & & & \\
\sigma_{3} \sigma_{1} \rho^{2} & \sigma_{3} \sigma_{2} \rho & \sigma_{3}^{2} & & & & \\
\sigma_{4} \sigma_{1} \rho^{3} & \sigma_{4} \sigma_{2} \rho^{2} & \sigma_{4} \sigma_{3} \rho & \sigma_{4}^{2} & & & \\
\sigma_{5} \sigma_{1} \rho^{4} & \sigma_{5} \sigma_{2} \rho^{3} & \sigma_{5} \sigma_{3} \rho^{2} & \sigma_{5} \sigma_{4} \rho & \sigma_{5}^{2} & & \\
\sigma_{6} \sigma_{1} \rho^{5} & \sigma_{6} \sigma_{2} \rho^{4} & \sigma_{6} \sigma_{3} \rho^{3} & \sigma_{6} \sigma_{4} \rho^{2} & \sigma_{6} \sigma_{5} \rho & \sigma_{6}^{2} & \\
\sigma_{7} \sigma_{1} \rho^{6} & \sigma_{7} \sigma_{2} \rho^{5} & \sigma_{7} \sigma_{3} \rho^{4} & \sigma_{7} \sigma_{4} \rho^{3} & \sigma_{7} \sigma_{5} \rho^{2} & \sigma_{7} \sigma_{6} \rho & \sigma_{7}^{2}
\end{array}\right]
$$

Eq. 4

O vetor de efeitos fixos $(\beta)$ foi estimado considerando a matriz de covariância Mínimos Quadrados Generalizados. Os componentes de variância foram estimados simultaneamente, utilizando o método de Máxima Verossimilhança Restrita (REML).

O vetor do parâmetro aleatório $(u)$ foi predito com base na Equação 5, considerado o me- lhor estimador para o preditor linear não enviesado (EBLUP-Empirical Best Linear Unbiased Predictor):

$$
\widehat{u}=\widehat{G} Z^{\prime} \widehat{V}^{-1}(y-X \hat{\beta})
$$

$$
\begin{gathered}
\text { sendo } \hat{V}^{-1}=\left(R+Z D Z^{\prime}\right)^{-1} \mathrm{e}(y-X \hat{\beta})=\varepsilon \mathrm{e}, \text { tem-se } \\
\hat{u}=\hat{G} Z^{\prime}\left(R+Z D Z^{\prime}\right)^{-1} \varepsilon
\end{gathered}
$$

Em que: $G=$ matriz diagonal; $Z=$ matriz de delineamento para o componente aleatório; $R=$ matriz da variância residual estimada (EQUAÇÃO 4), em que $R \neq \sigma^{2} l ; \varepsilon=$ vetor com dimensão correspondente ao número de observações em que os componentes são valores residuais.

O fator de inflação da variância (VIF) foi verificado para todas as variáveis preditoras incluídas no modelo fixo (CHATTERJEE et al., 2000). Testes de validação baseados nos dados observados e estimados foram calculados como parâmetros na avaliação da eficiência da predição do modelo misto (Tabela 2).

O pressuposto de normalidade e homogeneidade de variâncias foram avaliados utilizando os testes de Kolmogorov-Smirnov e Levene, respectivamente. Todas as análises estatísticas foram feitas com o sistema estatístico SAS V.9.1 (SAS/STAT, 
TABELA 2: Critérios para avaliação do modelo de regressão para AG.

TABLE 2: AG regression model performance evaluation criteria.

\begin{tabular}{|c|c|c|c|}
\hline Critério & Símbolo & Fórmula & Ideal \\
\hline Coeficiente de variação & $\mathrm{CV}$ & $\left\{\frac{\sum_{i=1}^{n}\left(o b s_{i}-e s t_{i}\right)^{2}}{n-p}\right\}^{1 / 2} / \overline{o b s_{l}}$ & 0 \\
\hline \multirow[t]{2}{*}{ Coeficiente de determinação } & $\mathrm{R}^{2}$ & $1-\left[\frac{\sum_{i=1}^{n}\left(o b s_{i}-e s t_{i}\right)^{2}}{\sum_{i=1}^{n}\left(o b s_{i}-\overline{o b s}\right)^{2}}\right.$ & \multirow[t]{2}{*}{1} \\
\hline & Critério & & \\
\hline Critério de Akaike corrigido & $(\mathrm{AICC})$ & & melhor \\
\hline Critério de Informação Bayesiano & (BIC) & & melhor \\
\hline
\end{tabular}

2004) no procedimento MIXED, com significância de $\mathrm{p}<0,05$.

\section{RESULTADOS E DISCUSSÃO}

O coeficiente de correlação de Pearson revelou correlação em série para os dados do alongamento da copa, conforme demonstrado na Figura 2 , indicando que o alongamento do ano " $i$ " depende de forma positiva do alongamento do ano "i-1".

Este tipo de autocorrelação, frequente em dados de crescimento, pode ocorrer em função da disponibilidade de água (precipitação no sítio), área de copa ou grau de infestação por lianas conforme relatam Brienen e Zuidema (2006).

Apesar da elevada variação, o AG está correlacionado ao incremento corrente anual em diâmetro em 53\% (correlação linear de Pearson; $\mathrm{p}=<0,0001)$. O modelo linear simples descreveu esse comportamento $(\mathrm{p}<0,0001)$, conforme a Figura 3. Estudos conduzidos por Durlo (2001) mostraram estreita relação entre o tamanho da copa com o incremento em diâmetro em árvores singulares - crescimento livre de concorrência - de cedro.

A relação linear positiva entre o crescimento da copa com o diâmetro do fuste foi verificado em árvores singulares em pesquisas anteriores (cf. HEMERY et al., 2005; PIBOULE et al., 2005), o que permitiu inferir que o incremento em diâmetro é uma variável potencial para descrever AG.

A análise da Figura 4 permitiu verificar que utilizar um modelo que assume ser iid $\mathrm{N}\left(0, \sigma^{2} l\right)$ seria claramente inadequado, pois o comprimento do galho ocorrido em anos consecutivos correlacionou-se exponencialmente com a magnitude do período (LAG) com variâncias heterogêneas (linha descontínua). Este resultado indicou a utilização da estrutura da matriz de covariância heterogênea e autorregressiva (ARH(1)) já que, de acordo com Littell et al. (2006), a inferência dos resultados oriundos dos efeitos fixos dependem da modelagem correta desta matriz.

De acordo com o modelo 1 (Tabela 3), árvores com a menor taxa de incremento corrente anual em diâmetro mostraram um alongamento médio da copa em torno de $10,6 \mathrm{~cm}$, aumentando a uma taxa de $4,8 \mathrm{~cm}$ por cada unidade de incremento anual ocorrido no diâmetro. Os componentes aleatórios associados ao intercepto (que expressa a variação do alongamento entre as diferentes classes de altura) e ângulo apresentaram erro padrão assintótico elevado tornando não significativas suas variâncias estimadas. Este fato pode não ser consistente por dois motivos: (1) para amostras pequenas (menor que 120 observações) a estimação do erro padrão é afetada e geralmente os componentes de variância possuem distribuição assimétrica tornado o teste de hipótese não realístico (SINGER, 1998); (2) o valor da variância para o intercepto é maior do que a metade do erro residual estimado, podendo indicar que parte da variação total do modelo não foi explicada.

Os coeficientes aleatórios para o intercepto e ângulo do modelo 2, para cada classe de altura total, (Tabela 4) foram definidos para corrigir os valores em nível e ângulo da equação de alongamento do galho dentro da classe de altura estabelecida.

Ao avaliar o efeito do tamanho inicial da árvore no comprimento do galho, o modelo incluiu o diâmetro (d) e altura comercial (hc) como variáveis significativas (Equação 2) resultando na redução do componente aleatório que descreve a variação em nível para 53,6\%, aumentando a precisão da estimativa em relação à linha média (Figura 5). 

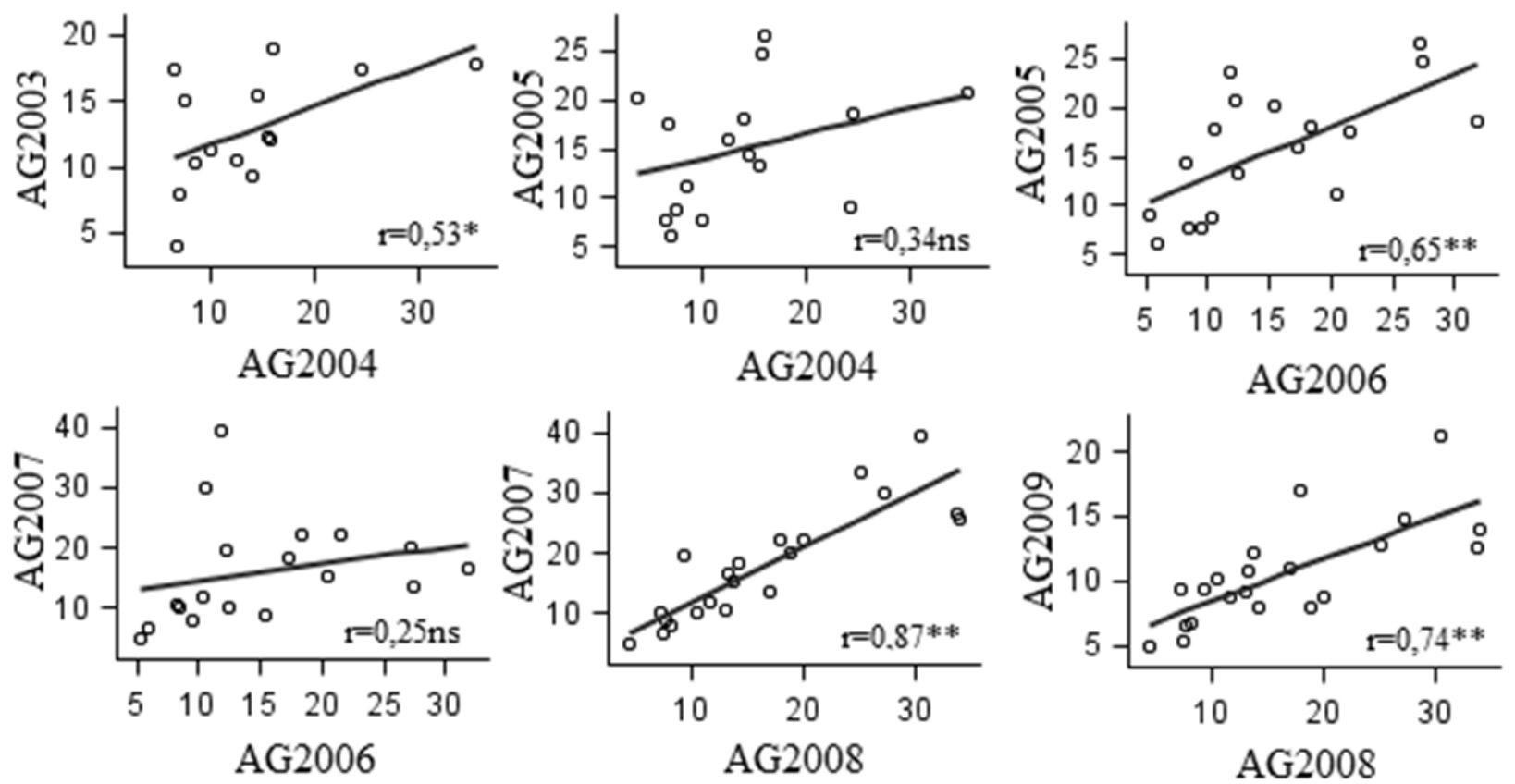

FIGURA 2: Relação do alongamento do galho $(\mathrm{cm})$ em anos consecutivos para nível de significância de $5 \%$.

FIGURE 2: Branch growth relationship $(\mathrm{cm})$ between consecutive years for $5 \%$ of significant level.

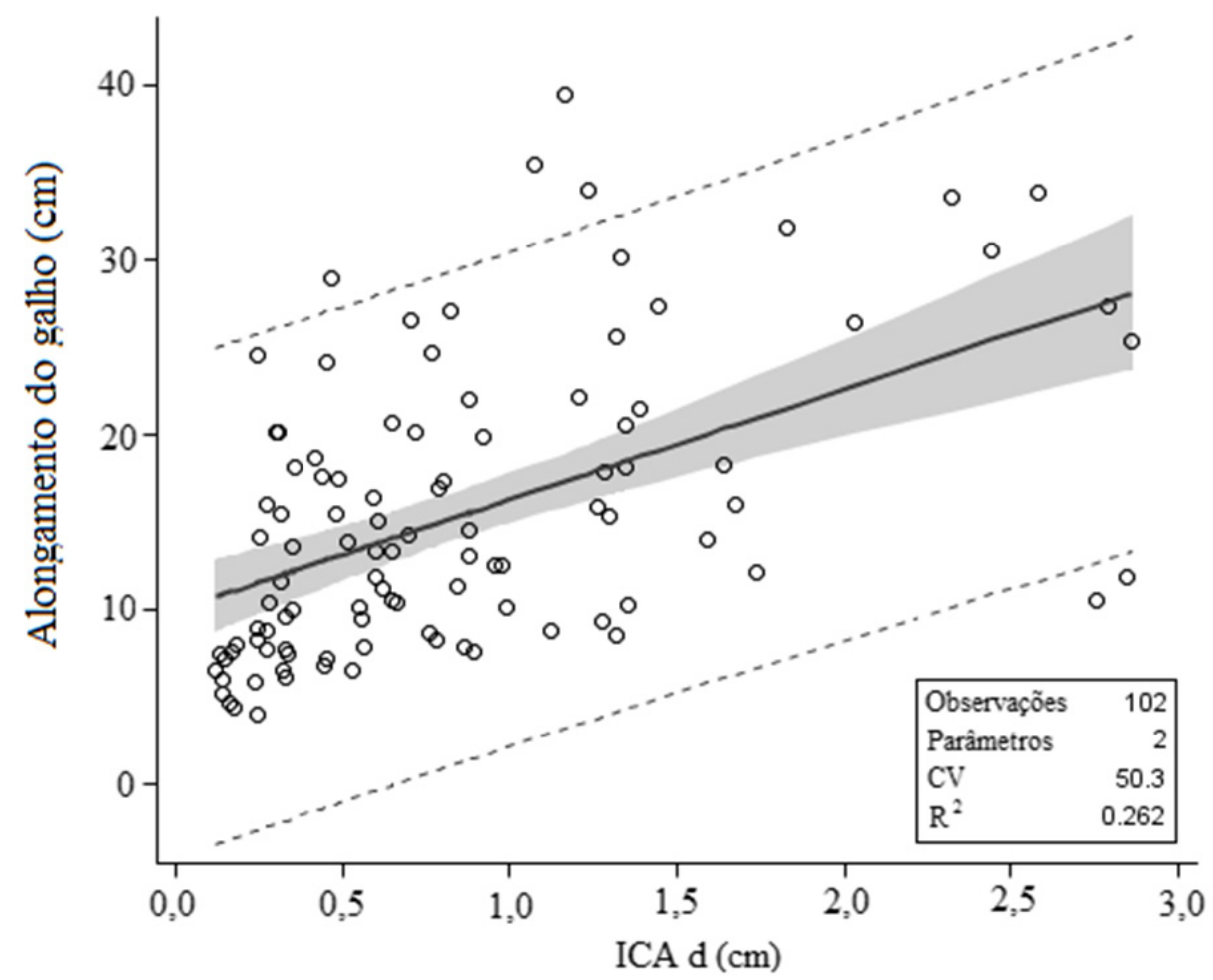

FIGURA 3: Alongamento anual do galho observado (o) e estimado (linha contínua) em função do incremento corrente anual em diâmetro. A área sombreada e a linha descontínua indicam os limites de confiança para a média e para a estimação, respectivamente.

FIGURE 3: Observed ( $\circ$ ) and estimated (filled line) annual crown growth as a function of current annual increment. The shaded area and the doted line denoted the confidence limit for the mean and for predicted values, respectively. 


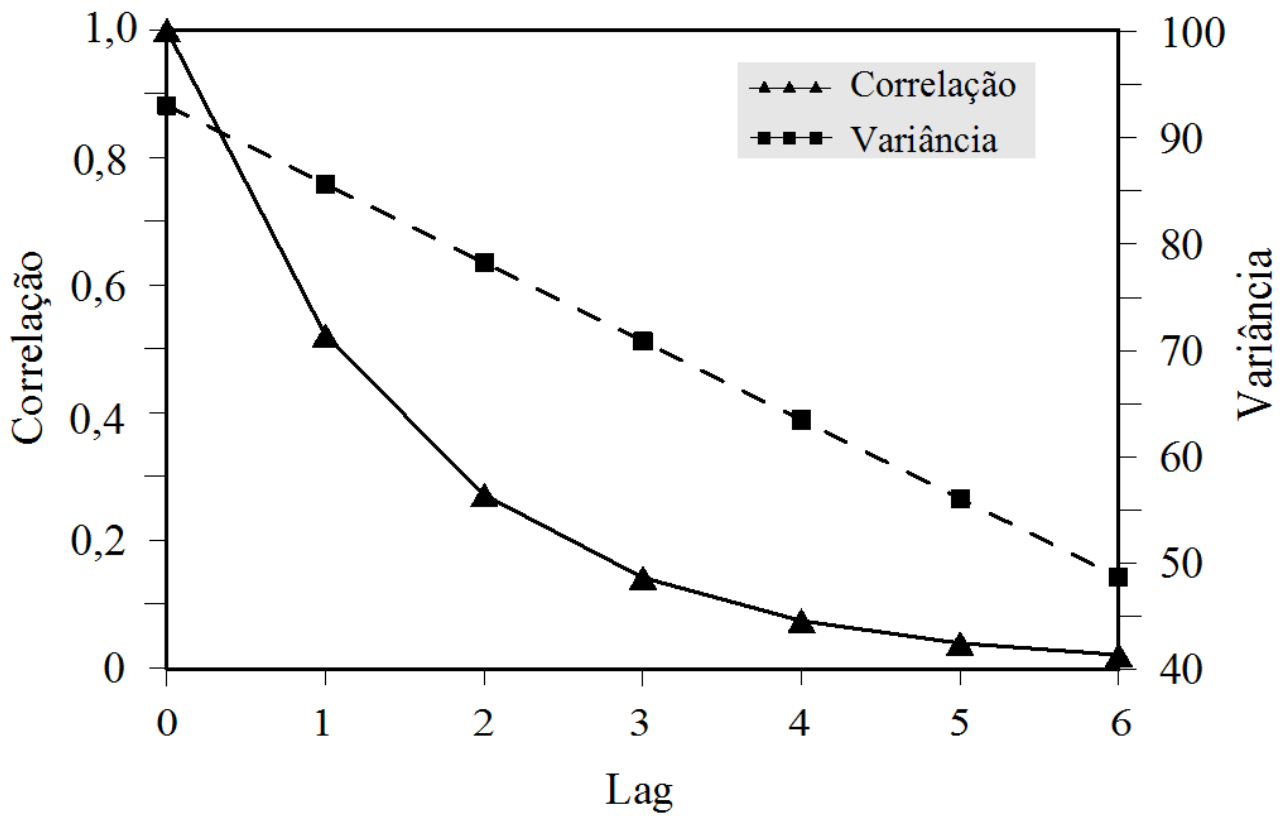

FIGURA 4: Correlação e variância residual do alongamento do galho no período avaliado. FIGURE 4: Correlation and residual variance to crown growth on the evaluated period.

TABELA 3: Variáveis preditoras e seu efeito no alongamento anual do galho e critérios de informação para avaliação.

TABLE 3: Predictors variables and its effect on crown growth and information criteria to evaluation.

\begin{tabular}{|c|c|c|c|c|}
\hline & \multicolumn{2}{|c|}{ Modelo 1} & \multicolumn{2}{|c|}{ Modelo 2} \\
\hline & Coeficiente (EP) & $\mathrm{p}$ & Coeficiente (EP) & $\mathrm{p}$ \\
\hline \multicolumn{5}{|l|}{ Efeito fixo } \\
\hline Intercepto & $10,5928(1,66)$ & $<0,0001$ & $6,3540(2,99)$ & 0,0362 \\
\hline$I C A d_{i j}$ & $4,7920(1,29)$ & 0,0013 & $3,6389(1,09)$ & 0,0461 \\
\hline$d_{i j}$ & & & $-0,4074(0,09)$ & $<0,0001$ \\
\hline \multirow[t]{2}{*}{$h c_{i j}$} & & & $1,8011(0,43)$ & $<0,0001$ \\
\hline & Variância (EP) & $\mathrm{p}$ & Variância (EP) & $\mathrm{P}$ \\
\hline \multicolumn{5}{|l|}{ Efeitos aleatórios } \\
\hline Nível 1 resíduos & 43,8971 & $<0,0001$ & 24,1369 & $<0,0001$ \\
\hline \multicolumn{5}{|l|}{ Nível 2} \\
\hline Intercepto $\left(u_{0 j}\right)$ & $29,4112(28,67)$ & 0,1525 & $13,6536(15,10)$ & 0,1830 \\
\hline Ângulo $\left(u_{l j}\right)$ & $7,4647(14,84)$ & 0,3076 & $13,1366(19,72)$ & 0,2526 \\
\hline $\mathrm{CV} \%$ & 38,6 & & 27,3 & \\
\hline $\mathrm{R}^{2}$ & 0,527 & & 0,698 & \\
\hline AICC & 707,6 & & 693,0 & \\
\hline BIC & 706,7 & & 671,0 & \\
\hline
\end{tabular}

$\mathrm{EP}=$ erro padrão assintótico. 
TABELA 4: EBLUPs preditos para corrigir o alongamento do galho em nível e taxa de alongamento do galho por classe de altura total.

TABLE 4: Predicted EBLUP's to estimate the level and rate of crown growth by total height class.

\begin{tabular}{|c|c|c|}
\hline Variável & $\begin{array}{l}\text { Classe de } \\
\text { Altura (m) }\end{array}$ & $\begin{array}{c}\text { EBLUPs } \\
\left(u_{j}\right)\end{array}$ \\
\hline $\begin{array}{c}\text { Intercepto } \\
\quad I C A d_{i j}\end{array}$ & $a 7 \vdash 9$ & $\begin{array}{l}0,2475 \\
1,0378\end{array}$ \\
\hline $\begin{array}{c}\text { Intercepto } \\
\qquad I C A d_{i j}\end{array}$ & $a 9+11$ & $\begin{array}{c}2,6062 \\
-3,4510\end{array}$ \\
\hline $\begin{array}{c}\text { Intercepto } \\
\quad I C A d_{i j}\end{array}$ & $a 11+13$ & $\begin{array}{c}-4,3478 \\
3,1433\end{array}$ \\
\hline $\begin{array}{c}\text { Intercepto } \\
\qquad I C A d_{i j}\end{array}$ & $a 13+17$ & $\begin{array}{r}2,4846 \\
-2,4161\end{array}$ \\
\hline $\begin{array}{c}\text { Intercepto } \\
\quad I C A d_{i j}\end{array}$ & $a 17 \vdash 20$ & $\begin{array}{c}-0,9906 \\
1,6859\end{array}$ \\
\hline
\end{tabular}

Em que: $7+9=$ coeficiente aleatório para a classe de altura compreendida entre 7 e 9 metros.

\section{Diagnóstico dos modelos}

A menor variação relativa captada pelo ajuste da Equação 2 justificou a inclusão das covariáveis diâmetro e altura comercial, como verificado pela sobreposição dos dados estimados sobre os observados (Figura 5a), levando a uma redução dos critérios de informação AICC e BIC (ver Tabela 3).

A análise residual destinada a avaliar os pressupostos de regressão e a qualidade da estimação obtida pela Equação 2, representada na Figura 6, mostrou um padrão aleatório da distribuição em função do valor estimado de AG (Figura 6a) para os resíduos studentizados condicionados (que considera o efeito aleatório). Além deste, foi possível comprovar o atendimento do pressuposto de distribuição normal (Figura 6b), confirmado pela não significância do teste de Kolmogorov-Smirnov. O gráfico de Box Plot revelou distribuição homogênea pelo teste de Levene dos resíduos ao longo das classes de altura total (Alongamento do galho constante ao longo das classes) demonstrado na Figura 6d também avaliado pela análise de variância. Este comportamento da distribuição dos resíduos demonstrou a correta seleção da estrutura do componente de variância.
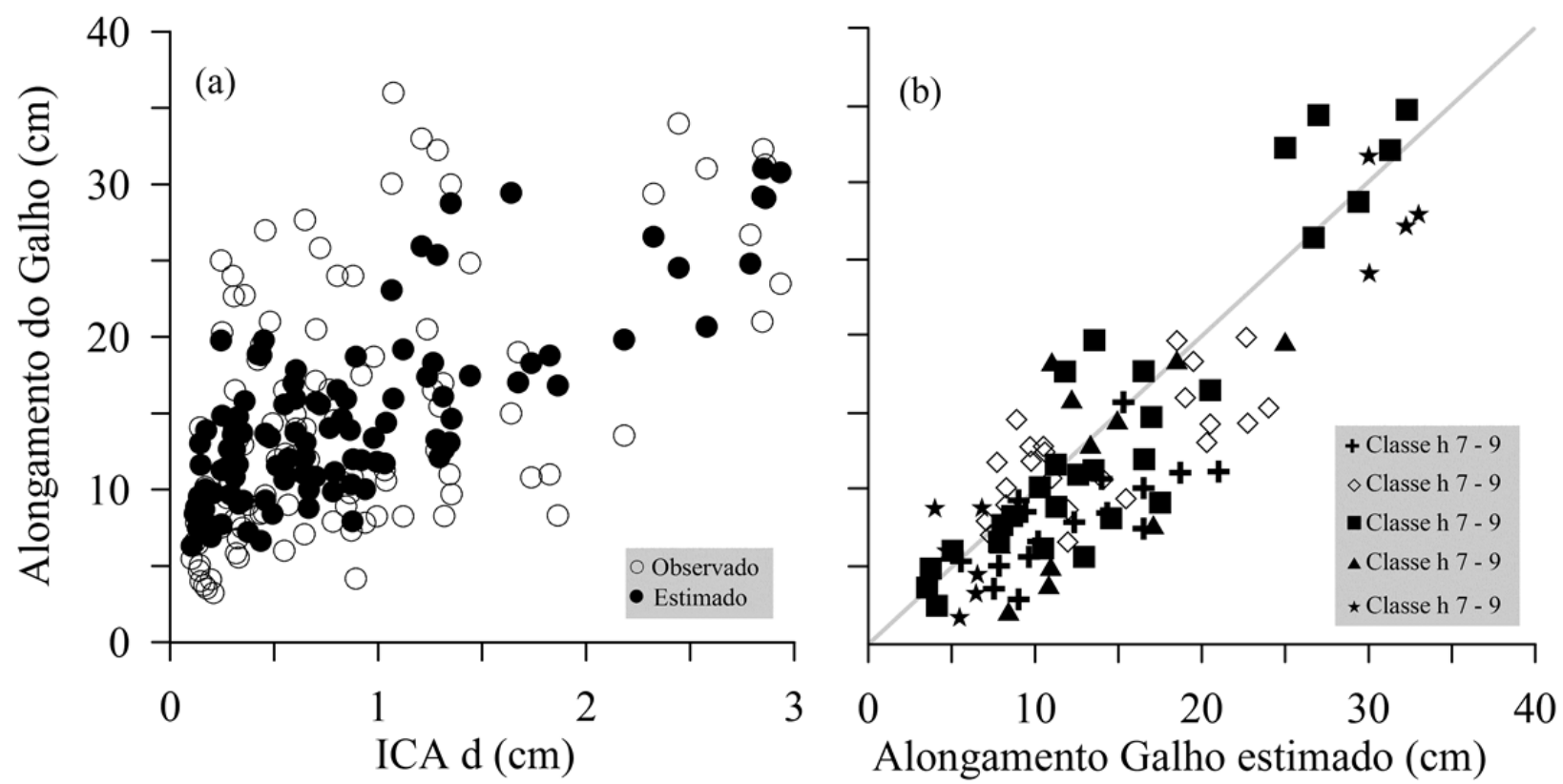

FIGURA 5: Comportamento do alongamento do galho para os anos de 2003 a 2009 representados em função do incremento corrente anual em diâmetro (a) e para cada classe de altura total (b).

FIGURE 5: Crown growth performance from 2003 to 2009 years as a function of diameter annual increment (a) by total height class (b). 

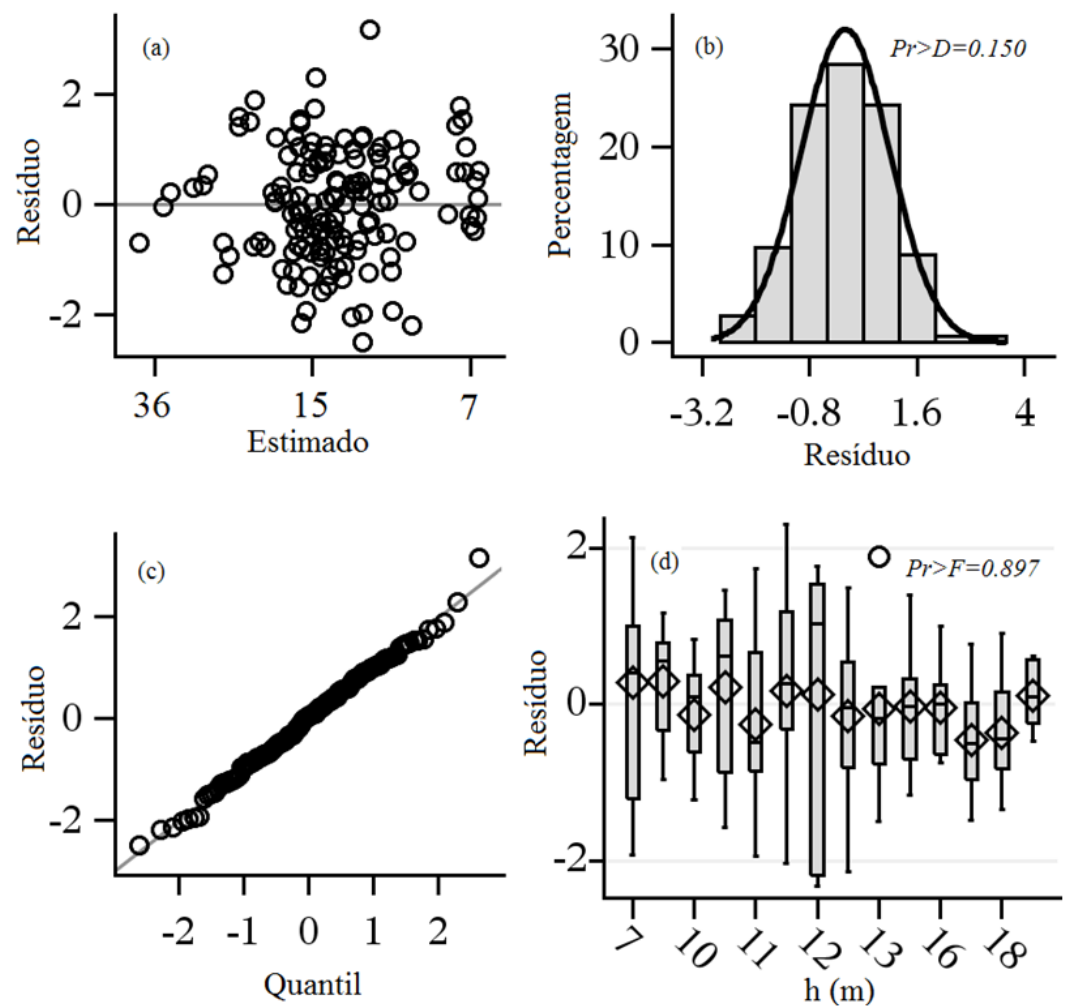

FIGURA 6: Distribuição de resíduos condicionados padronizados da Equação ajustada 2. FIGURE 6: Conditional standardized residual distribution from the fitted equation 2.

\section{CONCLUSÃO}

Examinando o processo do desenvolvimento da copa, representado pelo alongamento do galho (AG), conclui-se que, mudanças na expansão anual da copa, em árvores singulares de cedro, são acompanhadas por mudanças no crescimento anual em diâmetro, sendo esta, uma variável potencial para descrever o comportamento anual da copa.

Em decorrência da autocorrelação entre as medidas de alongamento do galho, o modelo linear misto melhor descreve a relação entre as variáveis, quando da inclusão de um componente aleatório para o intercepto e para o ângulo utilizando a matriz de covariância heterogênea e autorregressiva de primeira ordem.

O teste da razão de máxima verossimilhança revela ser altamente significativa a melhoria no ajuste dos modelos que incluem efeitos aleatórios e uso de matriz de covariância com estrutura especifica, quando da presença de dependência nos dados da variável dependente.

Por fim, o modelo permitiu quantificar o efeito do incremento diamétrico sobre o alongamento do galho possibilitando seu emprego como ferramenta de planejamento para atividades silviculturais da espécie.

\section{REFERÊNCIAS BIBLIOGRÁFICAS}

ACERBI, J. F. W. et al. Modelo para prognóse do crescimento e da produção e análise econômica de regimes de manejo para Pinus taeda. Revista Árvore, Viçosa, v. 26, p. 699-713, 2002.

BAPTISTA, V. R. Estudo da periodicidade do crescimento, fenologia e relação com a atividade cambial de espécies arbóreas tropicais de florestas estacionais semi-deciduais. 2002. 123 f. Dissertação (Mestrado em Recursos Florestais) - Escola Superior de Agricultura Luiz de Queiroz, 2002.

BRIENEN, R. J. W.; ZUIDEMA, P. A. Lifetime growth patterns and ages of Bolivian rain forest trees obtained by tree ring analysis. Journal of Ecology, v .94, p. 481-493, 2006.

BURGER, H. Baumkrone und zuwachs in zwei hiebsreifen fichtenbeständen. Mitteilungen der Schweizerischen Anstalt für das Forstliche Versuchswesen, v. 21, p. 147-176, 1939.

CHATTERJEE, S.; HADI, A. S.; PRICE, B. 
Regression analysis by examples. 3rd ed. New York: John Wiley \& Sons, 2000, p. 359.

DURLO, M. A.; DENARDI, L. Morfometría de Cabralea canjerana, em mata secundária nativa do Rio Grande do Sul, Ciência Florestal, v. 8, p. 55-66, 1998.

DURLO, M. A. Relações morfométricas para Cabralea canjerana (Well.) Mart. Ciência Florestal, v.11, p. 141- 149, 2001.

DURLO, M. A.; SUTILI, F. J.; DENARDI, L. Modelagem da copa de Cedrelafisilis Vellozo. Ciência Florestal, v. 14, p. 79-89, 2004.

EISFELD, R. L. et al. Modelagem do crescimento e da produção de Pinus taeda L. por meio do processo de difusão. Cerne, Lavras, v. 11, p. 167-177, 2005. HASENAUER, H.; MONSERUD, R. A. A crown ratio model for Austrian forest. Forest Ecology and Management, v. 84, p. 49-60, 1996

HEMERY, G. E.; SAVILL, P. S.; PRYOR, S. N. Applications of the crown diameter-stem diameter relationship for different species of broadleaved trees. Forest Ecology and Management, v. 215, p. 285-294, 2005.

LITTELL, R. C. et al. SAS for mixed models, 2nd ed. Cary, N. C.: SAS Institute Inc, 2006. 723 p.

MATTOS, P. P.; SEITZ, R. A.; MUÑIZ, G. I. B. Identification of annual growth rings base on periodical shoot growth. In: WIMMER, R.; VETTER, R. E. (Ed.) Tree ring analysis:biological, methodological and environmental aspects. Wallingford: CABI, 1999. p. 139-145.

MITCHELL, K. J. Dynamics and simulated yield of Douglas-fir. Forest Science Monography. p. 17-39. 1975.
NUTTO, L. Manejo do crescimento diamétrico de Araucária angustifólia (Bert.) O. Ktze. Baseadonaárvore individual, Ciência Florestal, v. 11, p. 9-25, 2001.

PIBOULE, A. et al. Reconstructing crown shape from stem diameter and tree position to supply light models. I. Algorithms and comparison of light simulations. Annals of Forest Science, v. 62, p. 645-657, 2005.

PRETZSCH, H. Konzeption und Konstruktion von Wuchsmodellen fur Rein- und Mischbestand. Forstliche Forschungsberichte Munchen, p. 115332, 1992.

PRETZSCH, H. Perspektiven einer modellorientierten Waldwachstumsforschung. Forstwissenschaftliches Centralblatt, v. 114, p. 188-209, 1995.

REMPHREY, W. R.; PEARN, L. P. Crown development of a clone of Populus tremuloides exhibiting "crooked" architecture and a comparison with wild-type trees. Canadian Journal of Botany, v. 81, p. 345-359, 2002.

RINN, F. TSAP-Win reference manual: computer program for tree ring analysis and presentation. Frank Rinn, Heidelberg, 2003, $263 \mathrm{p}$.

SAS/STAT. SAS Institute Inc., Cary, NC, 2004.

SINGER, J. Using SAS PROC MIXED to fit multilevel models, Hierarchical models, and Individual growth models. Journal of Education and Behavioral Statistics, v. 24, p.323-355, 1998. WORBES M. How to measure growth dynamics in tropical trees - a review. IAWA Journal, v. 16, p. 337-351, 1995. 